\title{
Direct Evidence for Block-by-Block Growth in High-Temperature Superconductor Ultrathin Films
}

\author{
M. Varela, ${ }^{1}$ W. Grogger, ${ }^{2}$ D. Arias, ${ }^{3, *}$ Z. Sefrioui, ${ }^{3}$ C. León, ${ }^{3}$ C. Ballesteros,${ }^{1}$ K. M. Krishnan, ${ }^{2}$ and J. Santamaría ${ }^{3, \dagger}$ \\ ${ }^{1}$ Departamento Física, Universidad Carlos III de Madrid, Avenida de la Universidad 30, 28911 Leganés, Madrid, Spain \\ ${ }^{2}$ Materials Sciences Division, 72-222, Lawrence Berkeley National Laboratory, University of California, Berkeley, California 94720 \\ ${ }^{3}$ GFMC, Departamento Física Aplicada III, Universidad Complutense, Ciudad Universitaria, 28040 Madrid, Spain
}

(Received 5 June 2000)

\begin{abstract}
Charge neutrality and stoichiometry impose severe restrictions on the mechanisms of epitaxial growth of complex oxides. The fundamental question arises of what is the minimum growth unit when sample thickness is reduced beyond the size of the unit cell. We have investigated the growth mechanism of $\mathrm{YBa}_{2} \mathrm{Cu}_{3} \mathrm{O}_{7}$ cuprate superconductor, using a consistent approach based on the growth of noninteger numbers of $\mathrm{YBa}_{2} \mathrm{Cu}_{3} \mathrm{O}_{7}$ layers in $\mathrm{YBa}_{2} \mathrm{Cu}_{3} \mathrm{O}_{7} / \mathrm{PrBa}_{2} \mathrm{Cu}_{3} \mathrm{O}_{7}$ superlattices. Ex situ chemical and structural analysis evidence a 2D block-by-block mechanism in which the minimum growth units are complete unit cell blocks, growing coherently over large lateral distances.
\end{abstract}

DOI: $10.1103 /$ PhysRevLett.86.5156

PACS numbers: 74.76.Bz, 61.10.Nz, 68.65.-k

The understanding of the microscopic processes involved in epitaxial growth and their impact on fundamental properties of ultrathin films has long been a central problem in surface and materials physics. For example, many fundamental studies of high- $T_{c}$ superconductors are based on measurements of ultrathin layers, where a one unit cell thick layer constitutes an attractive system to investigate dimensionality effects in vortex matter or the nature of high- $T_{c}$ superconductivity itself $[1,2]$. However, even though the possibility of growing a single unit cell coherently over large lateral distances has been debated in recent years, it still remains an open question [3,4]. In this context, since the physical properties of ultrathin layers might be seriously influenced by the specific growth mode [5], a major point of interest is to find the growth mechanism of complex oxides and the basic structural growth unit. Studies of the growth of ultrathin layers and understanding their growth modes require quantitative structural and chemical characterization at relevant length scales. Many studies fail because the chemical and physical disorder at the interfaces (interdiffusion and roughness) may invalidate the conclusions obtained for ultrathin layers [6]. In general, such disorder and its lateral length scale are largely determined by the particular growth mechanism. Moreover, the growth unit in complex ionic oxides is constrained to preserve charge neutrality and stoichiometry. This imposes a severe restriction on the layer-by-layer growth mechanism [7], a mode in which atomic layers are independently deposited and controlled. The alternative block-by-block growth mode [8] proposed for complex oxide thin films, in addition to preserving both charge neutrality and stoichiometry, also takes into account the thermodynamic driving forces present during the growth process at high substrate temperatures $\left(<700^{\circ} \mathrm{C}\right)$. According to this model, enough material must be available to form a whole unit cell (block) of the material. Diffusion on the hot substrate would provide the necessary mass transport for the layer to grow laterally in a 2D growth mode consisting of unit-cell-size blocks, coherently over large lateral distances. Various reports have been presented in recent years supporting both growth modes: Pennycook et al. [9] and Jia et al. [10] have shown the tendency of the system to complete unit cell blocks, however, more recently, Haage et al. [11] have claimed nonunit cell growth based on STM observations.

To resolve this long-standing but fundamental issue of growth modes, we present a consistent approach based on ex situ quantitative structural and chemical characterization of epitaxially grown ultrathin layers in $\mathrm{YBa}_{2} \mathrm{Cu}_{3} \mathrm{O}_{7-x} /$ $\mathrm{PrBa}_{2} \mathrm{Cu}_{3} \mathrm{O}_{7}$ (YBCO/PBCO) superlattices. From the analysis of superlattices with a noninteger number of $\mathrm{YBa}_{2} \mathrm{Cu}_{3} \mathrm{O}_{7-x}$ layers we provide concrete evidence for a two dimensional block-by-block growth mechanism in which the growth units are single YBCO unit cells and layer growth proceeds laterally, by the addition of one unit cell blocks.

$\mathrm{YBCO} / \mathrm{PBCO}$ superlattices were grown epitaxially on (100) $\mathrm{SrTiO}_{3}$ substrates held at a temperature of $900{ }^{\circ} \mathrm{C}$ in a high pressure (3.6 mbar pure oxygen) sputtering system. Such a high pressure oxygen atmosphere yields very thermalized growth at a very slow nominal growth rate of $0.013 \mathrm{~nm} / \mathrm{s}$ for YBCO which allows an accurate control of film thicknesses even below one unit cell $(\approx 1.2 \mathrm{~nm}$ for YBCO) [12]. High angle x-ray diffraction (XRD) spectra were analyzed using the SUPREX9 refinement software [13] which provides complete structural characterization of the superlattice along the growth direction, including layer thickness, and the disorder-related parameters, such as interdiffusion and interface roughness. Complementary, real space measurements of characteristic innershell excitations at high spatial resolution provide conclusive support for the chemical and structural details of the superlattices and their interfaces. These measurements are readily implemented in modern energy-filtering 
transmission electron microscopes (EFTEM) and, depending on the microscope parameters and the elements of interest, lateral resolution below $1 \mathrm{~nm}$ can be achieved [14-17]. All EFTEM investigations were performed using cross-section samples on a Philips CM200/FEG equipped with a field emission source and a post-column energy filter (Gatan Imaging Filter, GIF). We used the lowenergy edges of Pr and $\mathrm{Y}$ for imaging, applying the two window technique (jump ratio images, i.e., division of an image located right on the ionization edge by an image acquired before the edge onset) $[15,18]$. In this manner individual unit cells of $\mathrm{YBCO}$ and $\mathrm{PBCO}$ in the growth direction were differentiated, and individual $\{001\}$ atomic planes of $\mathrm{Y}$ and $\operatorname{Pr}$ were detected.

Typical XRD $(\theta-2 \theta)$ of a set of short period superlattices $\left[\mathrm{YBCO}_{n} / \mathrm{PBCO}_{5}\right]_{100 n m}$, with $n=1,1.2,1.5,1.7$, and 2 unit cells, are shown in Figs. 1(a)-1(e). The superlattice lateral coherence length, determined from the width of the superlattice Bragg peaks, was found to be around $80 \mathrm{~nm}$, showing that structural coherence is limited by sample thickness in the growth direction but it may be longer in the lateral dimension. Satellite peaks are determined by the modulation length $\Lambda$, with $\Lambda=N_{A} c_{A}+N_{B} c_{B}$, where $c_{A}$, $c_{B}, N_{A}$, and $N_{B}$ are the lattice parameters and number of unit cells of materials $A$ and $B$, respectively. Superlattice Bragg peaks are determined by the average lattice spacing $c=\Lambda /\left(N_{A}+N_{B}\right)$. In this way, diffraction peaks occur for $q$ vectors given by $q=2 \pi n / c \pm 2 \pi m / \Lambda$, where $n$ and $m$ are integers. Thus, if the modulation length is a noninteger number times the average lattice spacing, satellites from neighboring Bragg peaks will not be superimposed [19]. Moreover, in the presence of step disorder the noninteger modulation arises from a layer thickness fluctuation around a mean value, with a standard deviation quantifying the step-disorder roughness. This randomness causes satellite broadening and overshadows the apparent splitting in most cases. However, the analysis of the XRD patterns for an integer number of YBCO layers has shown the absence of step disorder in our superlattices [12]. Hence, in the absence of step disorder, the layer thickness does

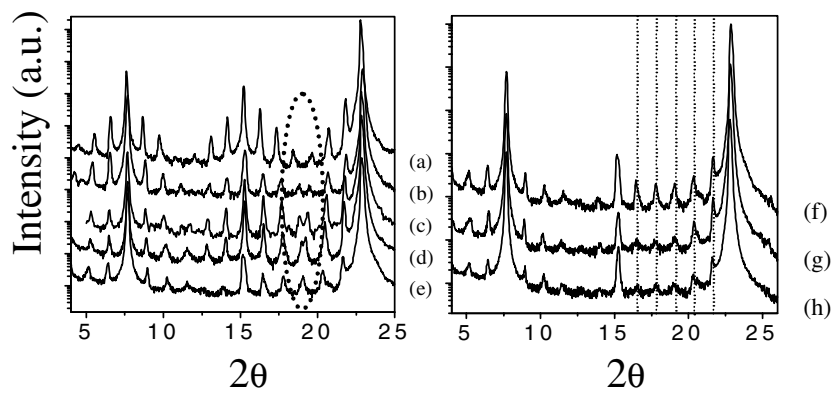

FIG. 1. $\theta-2 \theta$ spectra for a series of $\left[\mathrm{YBCO}_{n} / \mathrm{PBCO}_{5}\right]_{100 n m}$ superlattices with $n=2(a), 1.7(b), 1.5(c), 1.2(d)$, and $1(e)$. Note the nonmerging satellite peaks, encircled by a dotted line. Samples with $n=1(f), 0.5(g)$, and $0.2(h)$. Dashed lines show that superlattice modulation remains constant. not fluctuate in a random fashion, and an unusual superlattice diffraction pattern arises with sharp diffraction satellite peaks. Such a splitting in satellite orders is noticeable in Figs. 1(a)-1(e), confirming the controlled deposition of a noninteger number of layers, i.e., the composition changes coherently from interface plane to interface plane. Similar behavior has been previously observed in molecular beam epitaxially grown semiconductor superlattices [19] but this is the first time it has been observed in a complex oxide system. These $\mathrm{x}$-ray scattering results provide strong evidence for a lateral growth mode in which the growth units are complete YBCO units cells, i.e., a 2D block-by-block growth mechanism.

Further support for a block-by-block growth mechanism is obtained from XRD measurements of superlattices with YBCO layer thickness less than one unit cell. Figures 1(f)-1(h) show the XRD patterns of samples $\left[\mathrm{YBCO}_{n} / \mathrm{PBCO}_{5}\right]_{100 n m}$ with $n=0.2,0.5$, and 1 . Vertical lines show that the modulation length does not change and remains at the value $(7 \mathrm{~nm})$ corresponding to a strained $\left[\mathrm{YBCO}_{1} / \mathrm{PBCO}_{5}\right]_{100 \mathrm{~nm}}$ superlattice [12]. In other words, the repeating unit consists of a whole YBCO unit cell plus five PBCO cells, with the single YBCO cell not completely covering the substrate surface. In some sense the structure can be envisaged as a combination of $\mathrm{YBCO}_{1} / \mathrm{PBCO}_{5}$ and $\mathrm{YBCO}_{0} / \mathrm{PBCO}_{5}$ stacks, in which the $\mathrm{YBCO}_{0} / \mathrm{PBCO}_{5}$ stacks would not contribute to superlattice modulation but add disorder along the growth direction, thus reducing the satellite intensity. This finding points again to a 2D block-by-block growth mechanism where the growth of a nominal fraction of a unit cell implies the growth of a whole unit cell covering the corresponding fraction of the film surface.

Figure 2 shows individual jump ratio images for the $\left[\mathrm{YBCO}_{2} / \mathrm{PBCO}_{5}\right]_{100 \mathrm{~nm}}$ superlattice using the $\mathrm{Pr}-\mathrm{N}_{4,5}$ and $\mathrm{Y}-\mathrm{M}_{4,5}$ ionization edges together with the corresponding line profiles. In this superlattice, where the YBCO thickness is exactly an integer number, a perfectly smooth layer of uniform thickness can be observed. This demonstrates the ability of the energy-filtered imaging technique to image and resolve a unit cell thick layer of YBCO. Additionally, a certain amount of elastic contrast is always transferred to energy-filtered images [16] and hence, lattice planes can be seen in some of the jump ratio images. However, it should be pointed out that this contrast is not element specific.

A set of bright field images together with elementspecific energy-filtered images, using the characteristic $\operatorname{Pr}-\mathrm{N}_{4,5}$ edge for $\left[\mathrm{YBCO}_{n} / \mathrm{PBCO}_{5}\right]_{100 n m}$ superlattices, where $n=1.5$ and 0.2 is shown in Fig. 3. Each energyfiltered image [Figs. 3(b) and 3(d)] shows the stacking sequence of the two unit cells as well as the lateral coverage in a direction normal to the growth direction. Images of the superlattices with a noninteger number of unit cells ( $n<1$ and $1<n<2$ ) of YBCO in the stack (Fig. 3) show abrupt changes in the YBCO layer thickness from 

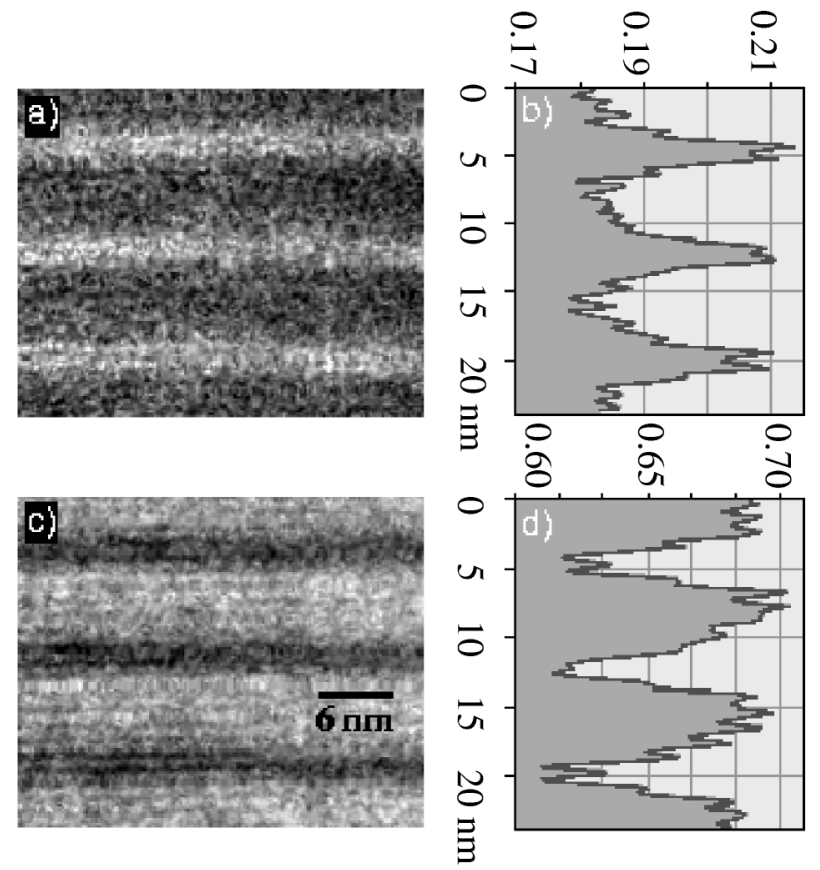

FIG. 2. Jump ratio images and corresponding line profiles using an integration width of 50 pixels for $\left[\mathrm{YBCO}_{2} / \mathrm{PBCO}_{5}\right]_{100 \mathrm{~nm}}$ : (a) jump ratio image using the $\operatorname{Pr}-\mathrm{N}_{4,5}$ ionization edge with (b) line profile across this image; (c) jump ratio image using the $\mathrm{Y}_{-\mathrm{M}_{4,5}}$ ionization edge with (d) line profile across this image. These images and their line profiles clearly show smooth YBCO layers of perfectly homogenous thickness.

one unit cell height, over a lateral length scale ranging from $100-200 \mathrm{~nm}$. The specimen thickness in the direction parallel to the electron beam is of the order of $10-30 \mathrm{~nm}$, well below the lateral dimension where a film of uniform thickness can be grown. For $n=1.5$ [Figs. 3(a) and 3(b)], the layer thickness of YBCO changes abruptly from one to two unit cells whereas for $n=0.2$ [Figs. 3(c) and 3(d)], the YBCO layers are clearly discontinuous and in cross section, appear as "islands" of one (and sometimes two) unit cell in height. The simultaneous presence of one unit cell high island provides a strong argument again for a block-by-block growth. Moreover, in both these cases the cumulative coverage of these laterally incomplete layers, experimentally measured from these images to be $\sim 1.26$ and $\sim 0.34$ unit cells of YBCO, respectively, is close to the average nominal composition of the superlattice. These chemically resolved energy-filtered images provide real space information at sufficient resolution to confirm the block-by-block growth mode.

Additional confirmation of this growth mode and the large lateral continuity of the layers are obtained from resistivity measurements. Although it is known that five PBCO unit cells are enough to prevent coupling through the PBCO layers [20], to be on the safe side we have grown another series of samples with PBCO spacers 10 unit cells thick. Figure 4 shows the resistance versus temperature characteristics of some typical $\left[\mathrm{YBCO}_{n} / \mathrm{PBCO}_{10}\right]_{100 \mathrm{~nm}}$

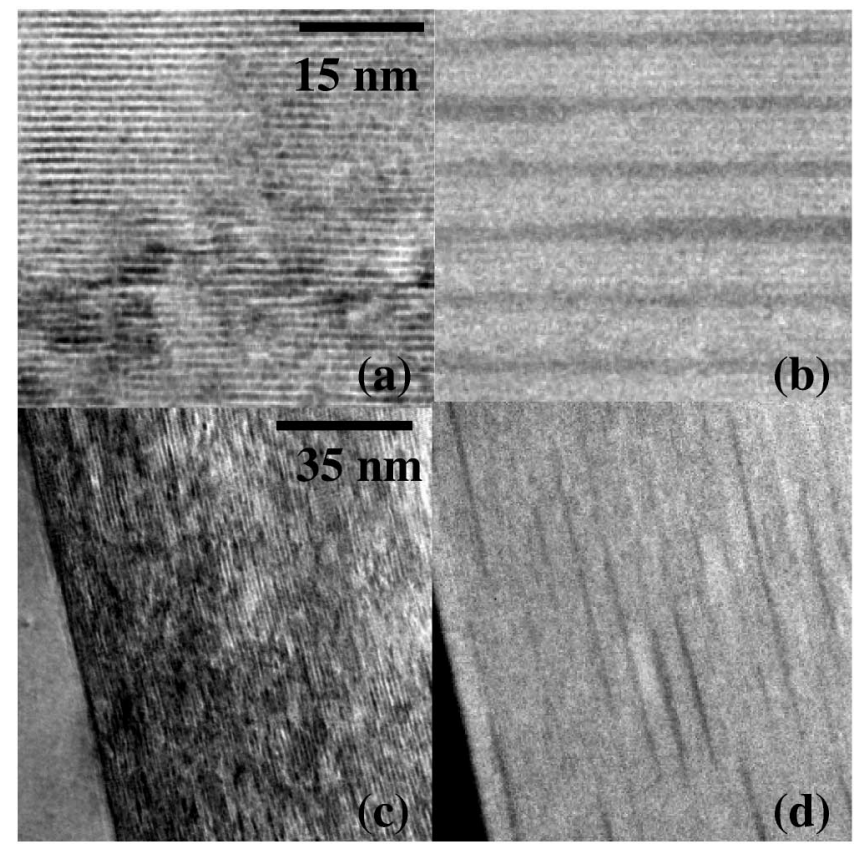

FIG. 3. TEM bright field and EFTEM images of $\left[\mathrm{YBCO}_{n} /\right.$ $\left.\mathrm{PBCO}_{5}\right]_{100 \mathrm{~nm}}$ samples in the cross-section view: (a) bright field and (b) jump ratio images using the $\operatorname{Pr}-\mathrm{N}_{4,5}$ ionization edge for $n=1.5$, (c) bright field and (d) jump ratio images using the Pr- $\mathrm{N}_{4,5}$ ionization edge for $n=0.2$. The elemental distribution map in (b) clearly shows YBCO layer thicknesses of one or two unit cells and well-defined steps where layer thickness changes. Additionally, no intermixing of $Y$ and $\operatorname{Pr}$ can be found. Between some of the PBCO layers in (d) "islands" of YBCO can be seen, obviously indicating the absence of any randomly distributed single unit cells of YBCO.

samples. The $\left[\mathrm{YBCO}_{1} / \mathrm{PBCO}_{10}\right]_{100 n m}$ superlattice shows superconducting behavior, with zero resistivity, $T_{c}$ of $40 \mathrm{~K}$. For $n<1$, the resistance-temperature data still show a superconducting transition for the $\left[\mathrm{YBCO}_{0.5} /\right.$ $\left.\mathrm{PBCO}_{10}\right]_{100 \mathrm{~nm}}$ sample and a hump at $T=60 \mathrm{~K}$ for the $\left[\mathrm{YBCO}_{0.3} / \mathrm{PBCO}_{10}\right]_{100 \mathrm{~nm}}$ sample, suggesting at least a superconducting onset. The superconducting behavior of such samples can be explained only by the picture of a distribution of islands one YBCO cell thick. In these laterally incomplete layers, electrical properties are shown to be extremely sensitive to the degree of substrate coverage of the superconducting layer. This is a critical aspect to be considered when debating experimental data on the transport properties of YBCO layers nominally one unit cell thick. Unfortunately, this has never been taken into account until now. Moreover, the signature of the coupling between such islands on the resistance curves should open a new field of research as a new way of creating weak links.

In conclusion, controlled growth of superlattices with a noninteger number of layers provides direct evidence for the 2D block-by-block growth of complex oxide thin films. Evidence is obtained from x-ray analysis of incommensurate superlattices showing coherent roughness. 


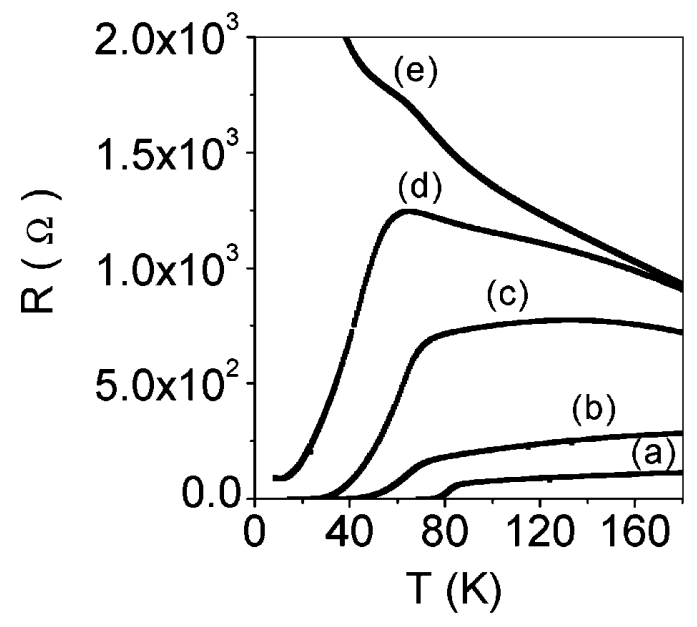

FIG. 4. Resistance versus temperature data for a series of $\left[\mathrm{YBCO}_{n} / \mathrm{PBCO}_{10}\right]_{100 n m}$ samples with $n=2(a), n=1(b)$, $n=0.8(c), n=0.5(d)$, and $n=0.3(e)$.

Complementary, chemically resolved energy-filtered images with nanometer scale resolution unambiguously confirm the block-by-block growth mode. These findings give direct indication that the structural growth unit in these oxides consists of a complete cuprate unit cell.

M. V. was partially supported by a research grant of the Fundación Universidad Carlos III de Madrid. Financial support from CICYT Grant No. MAT99-1706E is also acknowledged. M. V. is thankful for the hospitality received during her stay at the LBNL/NCEM. W. G. acknowledges support from the Max Kade Foundation for his stay at Berkeley. Work at LBNL/NCEM was supported by the Director, Office of Energy Research, Office of Basic Energy Sciences, Materials Sciences Division of the U.S. Department of Energy under Contract No. DE-AC03-76SF00098.
*On leave from Universidad del Quindio, Armenia, Colombia.

${ }^{\dagger}$ To whom all correspondence should be addressed. Email address: jacsan@eucmax.sim.ucm.es

[1] C. Dekker, P. J. M. Wöltgens, R. H. Koch, B. W. Hussey, and A. Gupta, Phys. Rev. Lett. 69, 2717 (1992).

[2] T. Terashima et al., Phys. Rev. Lett. 67, 1362 (1991).

[3] I. N. Chan et al., Phys. Lett. A 175, 241 (1993).

[4] J. Hasen, D. Lederman, and I. K. Schuller, Phys. Rev. Lett. 70, 1731 (1993).

[5] I. K. Schuller, Nature (London) 394, 419 (1998).

[6] E. E. Fullerton, J. Guimpel, O. Nakamura, and I. K. Schuller, Phys. Rev. Lett. 69, 2859 (1992).

[7] I. Bozovic and J. N. Eckstein, MRS Bull. 20, 32 (1995).

[8] J. P. Locquet, A. Catana, E. Mächler, C. Gerber, and J. G. Bednorz, Appl. Phys. Lett. 64, 372 (1994).

[9] S. J. Pennycook et al., Phys. Rev. Lett. 67, 765 (1991).

[10] C. L. Jia et al., Physica (Amsterdam) 210C, 1 (1993).

[11] T. Haage et al., Appl. Phys. Lett. 68, 2427 (1996).

[12] M. Varela et al., Phys. Rev. Lett. 83, 3936 (1999).

[13] E.E. Fullerton, I. K. Schuller, H. Vanderstraeten, and Y. Bruynseraede, Phys. Rev. B 45, 9292 (1992).

[14] O.L. Krivanek, M. K. Kundmann, and K. Kimoto, J. Microsc. 180, 277 (1995).

[15] F. Hofer, W. Grogger, G. Kothleitner, and P. Warbichler, Ultramicroscopy 67, 83 (1997).

[16] T. Navidi-Kasmai and H. Kohl, Ultramicroscopy 81, 223 (2000).

[17] Energy-Filtering Transmission Electron Microscopy, edited by L. Reimer (Springer, New York, 1995).

[18] O. L. Krivanek, A. J. Gubbens, M. K. Kundmann, and G. C. Carpenter, in Proceedings of the Annual Meeting of the Microscopy Society of America, edited by G. W. Bailey and C.L. Rieder (San Francisco Press, San Francisco, 1993), p. 586.

[19] I. K. Schuller et al., Phys. Rev. Lett. 65, 1235 (1990).

[20] J. M. Triscone and O. Fischer, Rep. Prog. Phys. 60, 1673 (1997). 